\title{
CAR-T cell Therapy: Past Successes and Future Challenges
}

\author{
Shruti Thakur and Rachana* \\ Department of Biotechnology, Jaypee Institute of Information Technology, India
}

Submission: April 03, 2018; Published: April 19, 2018

"Correspondence Address: Rachana, Department of Biotechnology, Jaypee Institute of Information Technology, Sector 62, Noida, UP, India, Email: rachana.dr@iitbombay.org

\section{Introduction}

Cancer therapy in the last few decades has gone through immense changes in the treatment regimen. For the last years, the main formof treatment for any type of cancer were surgery, radiation and chemotherapy. These methods though the backbone of any cancer treatment do not help in advanced and relapsed cancer stages [1]. To develop therapies that could help patients in advanced stages of cancer, researchers developed targeted therapy in the form of monoclonal antibodies, and these were marketed for different types of cancer depending on the target they attack [2]. Some examples include imatinib (Gleecev) and trastuzumab (Herceptin), to name two. These drugs worked by honing in on specific molecular targets on cancer cells, becoming the standard of treatment for those cancers.

Over the last few years, though, immunotherapy has been gaining more interest as they work to activate the immune system for attacking the cancer cells. These therapies include pembrolizumab (Keytruda), nivolumab (Opdivo), and rituximab (Rituxan), among others [3]. Apart from these, a rapidly emerging therapy for activating immune response against cancer cells is adoptive cell transfer technique, which involves the extraction of patients' cells and reengineers them to equip them to treat their own cancer [4]. There have been many therapies in development as Adoptive T-cell Therapies, such as tumor-infiltrating lymphocytes (TILs) and T-cells transduced with high-affinity T-cell receptors (TCRs), but the therapy that has progressed from developmental phase to market is Chimeric Antigen Receptor (CAR) therapy [5].

CARs or Chimeric Antigen Receptors are receptors that have been engineered to create a specific immune effector cell ( $\mathrm{T}$ cell), and are made up of three parts: extracellular antigen recognition domain which is a variant of Single-chain fragment ( $\mathrm{scFv}$ ) that has been derived from an antibody, an intracellular $\mathrm{T}$ cell activated domain and a transmembrane domain [6]. These receptors, when engineered through patient's $\mathrm{T}$ cells, work to specifically target tumor cells. Out of all malignancies, CAR $\mathrm{T}$ cell therapy has shown greatest promise in hematological malignancies.

As per the records of end of 2016, out of the total clinical trials in oncology domain, 220 clinical trials were being conducted to develop CAR T. Even though CAR T was being developed for many years, the main breakthrough was observed in CAR T cells with CD19 specificity for B-cell malignancies [7]. This breakthrough exploded research efforts in this domain and the number of clinical trials rose exponentially. Until this point, CAR T cell therapy was of little interest to pharmaceutical companies as they preferred mass produced drugs and did not prefer researching individually tailored medicines. However, this changed with the successful results of clinical trials, and pharmaceutical companies went on to collaborate and partner with scientists and research groups developing this therapy [8].

Novartis teamed up with University of Pennsylvania, Kite Pharma with National Cancer Institute and Juno Therapeutics with Sloan Kettering [9]. These three companies were the forerunners of CAR $\mathrm{T}$ cell therapy development for human clinical use with their candidates, CTL019 (Kymriah), KTE-C19 (Yescarta), and JCAR015 being developed for Acute Lymphocytic Leukemia (ALL), Non-Hodgkin Lymphoma (NHL) and Acute Lymphocytic Leukemia (ALL), respectively. This CAR T therapy created a landmark in 2017 in the field of oncology, as FDA approved first 2 therapies that targeted CD19 [10]. These were Kymriah (tisagenlecleucel-T) from Novartis and Yescarta (axicabtageneciloleucel) from Kite Pharma/Gilead Sciences.

The former was approved for patients in pediatric settings who were suffering from relapsed or refractory B cell acute lymphoblastic leukaemia (ALL) and the latter was approved for Diffuse Large B cell lymphoma as well as some other NHL subtypes [11]. Kymriah's approval was a landmark on its own as its approval came in just six months after the US Food and Drug 
Administration had accepted its Biologic Licensing Application. As this application was taken up through priority review, the approval came through based on the clinical trial results of the ELIANA trial (phase II). In this trial, 83\% patients witnessed complete remission or complete remission with incomplete recovery of blood count [12]. As of now, the novel therapy is under consideration for aggressive NHL type Diffuse Large B cell Lymphoma (DLBCL), follicular lymphoma, multiple myeloma, and chronic lymphocytic leukaemia (CLL) in US and relapsed and refractory ALL and DLBCL in Europe.

The second therapy of CAR that was awarded FDA approval was Yescarta in October 2017, mainly for patients that had relapsed or refractory cell lymphomas, such as DLBCL and other NHL subtypes. The patients can take Yescarta after they have had at least two systemic therapies. The therapy is still under review in Europe [13]. The FDA approval was mainly due to the results of its ZUMA- 1 trial that included a single arm treatment group, where the results showed that $82 \%$ patients achieved Overall Response Rate (ORR) as well as $58 \%$ patients showed Complete Response (CR). These results were promising and showed that Yescarta could be effectively used in relapsed/refractory patients of ALL and other NHL subtypes. The therapy is currently being reviewed for Mantle Cell Lymphoma (MCL) as well as, Follicular Lymphoma under the FDA. For its already approved indications, FDA had granted it Priority Review as well asOrphan Drug and Breakthrough Therapy designations.

These CAR T cell therapies do not come without their adverse events and side effects associated. Kymriah has been provided with Black Box warnings for cytokine release syndrome (CRS), an event where there is an activation of CAR T cells and their proliferation, causing fever and flu like symptoms. Also, the therapy causes some neurological adverse events [14]. Both cytokine release syndrome and neurological events might be life threatening to patients who have taken Kymriah. Other moderately serious events include hypotension, infections, acute kidney disease, hypoxia and fever. These symptoms may appear as early as one day to 22 days after Kymriah administration. Also, another aspect that needs attention is that these engineered T cells may kill natural B cells of the patient's body, leading to increased risk of infections due to lack of antibodies. In terms of Yescarta, its Black Box also displays warning of CRS, and this therapy can also cause side effects such as low count of blood cells, weakened immune system and infections related to blood [15]. The time duration of contracting these side effects may range from 1-22 days, as is with the case of Yescarta.

In terms of availability, both Kymriah and Yescarta are available through restricted programs where patients need to undergo a risk evaluation as well as mitigation strategy process, wherein the adverse events are addressed related to CRS, neurotoxicity and other life-threatening adverse events. Furthermore, as these therapies are first of their kind and have been approved through an accelerated process, the regulatory agencies (especially FDA) require conduction of post marketing studies to evaluate the long term efficacy as well as long term risks and secondary malignancy chances associated with them . Not only this, the drug cost of these two therapies is another problem as Kymriah's regimen for pediatric patients runs at $\$ 475,000$, while Yescarta's adult regimen costs $\$ 373,000$.

Apart from side effects, adverse events and cost of these therapies, there is a need to assess the success of CARs in solid tumors, as the failure may results from a lack of any unique or specific tumor antigen in most cancers, the lack of ability of CAR cells to proliferate and persist after adoptive transfer, heterogeneous target antigen expression which leads to outgrowth of negative antigen tumor variants, as well as, insufficient amount of CAR T cells presence in tumor sites and metabolically hostile microenvironment of the tumor. Optimization of these therapies through biotechnological aspects can help overcome these limitations, and with time, pipeline for CAR-T therapies is expected to boom and reach beyond hematological cancers and deliver value therapeutically to solid tumor patients. It can be noted that this therapy's development is in its infancy stage and there is an uncertainty of how much commercial value this class can provide as off-the-shelf and solid tumor targeting form.

\section{References}

1. Yauch R, Settleman J (2012) Recent advances in pathway-targeted cancer drug therapies emerging from cancer genome analysis. Curr Opin Genet Dev 22(1): 45-49.

2. Antonio Di Stasi, Siok-Keen Tey, Gianpietro Dotti, Yuriko Fujita, Alana Kennedy-Nasser, et al. (2011) Inducible Apoptosis as a Safety Switch for Adoptive Cell Therapy. New England Journal of Medicine 365(18): 1673-1683.

3. Stegmeier F, Warmuth M, Sellers WR, Dorsch M (2010) Targeted Cancer Therapies in the Twenty-First Century: Lessons from Imatinib. Clin Pharmacol Ther 87(5): 543-552.

4. Yauch R, Settleman J (2012) Recent advances in pathway-targeted cancer drug therapies emerging from cancer genome analysis. Curr Opin Genet Dev 22(1): 45-49.

5. Bridgeman J, Hawkins RE, Hombach AA, Abken H, Gilham DE (2010) Building better chimeric antigen receptors for adoptive $\mathrm{T}$ cell therapy. Curr Gene Ther 10(2): 77-90.

6. Kalos M, June CH (2013) Adoptive $\mathrm{T}$ cell transfer for cancer immunotherapy in the era of synthetic biology. Immunity 39(1): 49-60.

7. Flotte TR (2018) Gene and Cell Therapy in 2018: A Look Ahead. Hum Gene Ther 29(1): 1-1.

8. Wilson JM (2017) 2017 Was the Year We Have Been Waiting For. Human Gene Therapy Clinical Development 28(4): 165-166.

9. Philippidis A (2017) Kymriah, First CAR-T Cancer Immunotherapy Approved by FDA. Clinical OMICs 4(5): 8.

10. Grady D (2016) FDA approves first gene-altering leukemia treatment, costing $\$ 475,000$. Image.

11. Prasad V (2017) Immunotherapy: Tisagenlecleucel - the first approved CAR-T-cell therapy: implications for payers and policy makers. Nat Rev Clin Oncol 15(1): 11-12. 


\section{Cancer Therapy \& Oncology International Journal}

12. Yauch R, Settleman J (2012) Recent advances in pathway-targeted cancer drug therapies emerging from cancer genome analysis. Curr Opin Genet Dev 22(1): 45-49.

13. Bonifant CL, Jackson HJ, Brentjens RJ, Curran KJ (2016) Toxicity and management in CAR T-cell therapy. Mol Ther Oncolytics 3: 16011.

This work is licensed under Creative Commons Attribution 4.0 License

DOI: 10.19080/CTOIJ.2018.10.555783
14. Yip A, Webster RM (2017) The market for chimeric antigen receptor T cell therapies. Nat Rev Drug Discov 17(3): 161.

15. D’Aloia M, Zizzari I, Sacchetti B, Pierelli L, Alimandi M (2018) CAR-T cells: the long and winding road to solid tumors. Cell Death \& Disease 9(3): 282.

Your next submission with Juniper Publishers will reach you the below assets

- Quality Editorial service

- Swift Peer Review

- Reprints availability

- E-prints Service

- Manuscript Podcast for convenient understanding

- Global attainment for your research

- Manuscript accessibility in different formats

(Pdf, E-pub, Full Text, Audio)

- Unceasing customer service

Track the below URL for one-step submission https://juniperpublishers.com/online-submission.php 